\title{
Десорбция водорода из пентаграфана
}

\author{
() Л.А. Опенов, А.И. Подливаев \\ Национальный исследовательский ядерный университет „МИФИ“, \\ 115409 Москва, Россия \\ ฯ E-mail: LAOpenov@mephi.ru
}

(Поступила в Редакцию 22 ноября 2018 г.

В окончательной редакции 10 декабря 2018 г.

Принята к публикации 17 декабря 2018 г.)

Путем атомистического компьютерного моделирования исследована термоактивированная десорбция водорода из пентаграфана - недавно предсказанного квазидвумерного углеводородного соединения, представляющего собой покрытый с обеих сторон водородом монослой пентаграфена, в котором связи С-C образуют сетку из смежных пятиугольников, а характерные для углеродных наноструктур шестиугольники отсутствуют. Изучено влияние десорбции водорода на электронную структуру, плотность фононных состояний и модуль Юнга. Методом молекулярной динамики определена температурная зависимость характерного времени десорбции.

DOI: $10.21883 /$ FTP.2019.05.47571.9029

\section{1. Введение}

Основным элементом структуры многих низкоразмерных углеродных материалов (фуллеренов [1], нанотрубок [2], графена [3]) являются шестиугольники из связей $\mathrm{C}-\mathrm{C}$. В классических фуллеренах присутствуют также пятиугольники. В графене и нанотрубках пятиугольники появляются при образовании дефектов Стоуна-Уэльса [4]. Наличие пятиугольников с общими сторонами энергетически невыгодно [5]. Тем не менее расчеты из первых принципов свидетельствуют о возможности существования пентаграфена - аллотропа углерода, состоящего только из пятиугольников [6].

Отличительной особенностью пентаграфена является присутствие в образце атомов углерода с различной гибридизацией электронных орбиталей: $s p^{3}$ (атомы C1) и $s p^{2}$ (атомы C2). Число ближайших соседей у этих атомов равно соответственно четырем и трем (см. рис. 1). Длина связи $d(\mathrm{C} 2-\mathrm{C} 2)=1.34 \AA$ существенно меньше длины связи $d(\mathrm{C} 1-\mathrm{C} 2)=1.55 \AA[6]$. Атомы С2 образуют взаимно перпендикулярные димеры и расположены в двух параллельных плоскостях, каждая из которых на $0.6 \AA$ отстоит от находящейся между ними плоскости атомов C1. Примитивная элементарная ячейка включает шесть атомов (два атома С1 и четыре атома С2).

Энергия связи пентаграфена выше, чем у графена $[7,8]$, т.е. с термодинамической точки зрения он неустойчив. Это служит одним из оснований для заключения о том, что его нельзя изготовить экспериментально [7]. Остается возможность существования пентаграфена в метастабильном состоянии, подобно фуллерену $\mathrm{C}_{20}[9,10]$. Действительно, данные ab initio молекулярной динамики свидетельствуют о том, что пентаграфен сохраняет свою структуру при конечной температуре, хотя и в течение весьма непродолжительного времени [6]. Оказывается, однако, что термоактивированный поворот димеров С2 инициирует образование дефектных участков, которые не остаются локализованными, а быстро распространяются на весь образец, приводя к его полному разрушению [11]. Еще одним препятствием на пути изготовления пентаграфена может стать сильный изгиб образцов в процессе роста и даже их сворачивание в пентатрубки под действием механических напряжений, создаваемых двумя подрешетками димеров С2 [12].

Для стабилизации структуры пентаграфена в работе [13] было предложено использовать его двустороннее гидрирование, в результате которого образуется пентаграфан (рис. 2), названный так авторами по аналогии с графаном - полностью гидрированным графеном [14]. Согласно расчетам [13], энергия формирования пентаграфана меньше, чем у других углеводородных соединений с такой же стехиометрией $\mathrm{C}: \mathrm{H}$, включая не полностью гидрированный графан. Из этого авторы

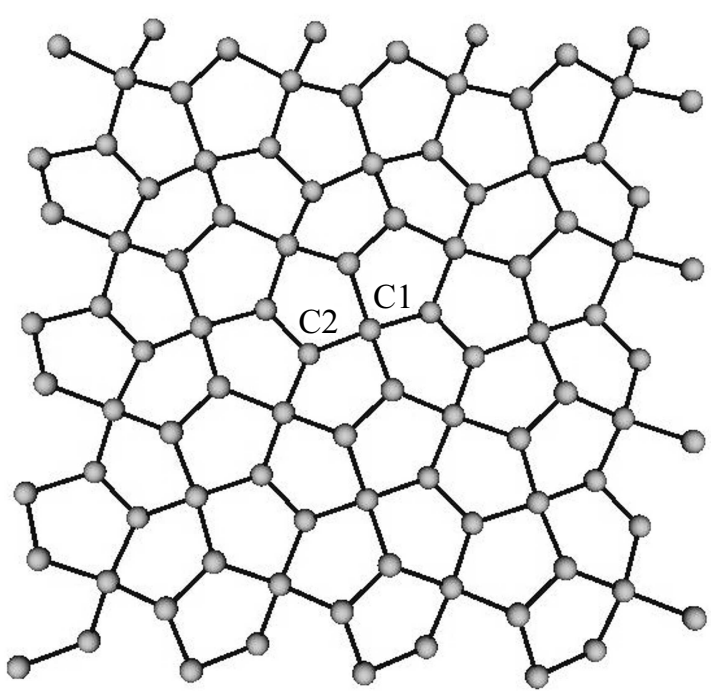

Рис. 1. Структура пентаграфена. $\mathrm{C} 1-s p^{3}$-гибридизованные атомы, C2-s $p^{2}$-гибридизованные атомы 


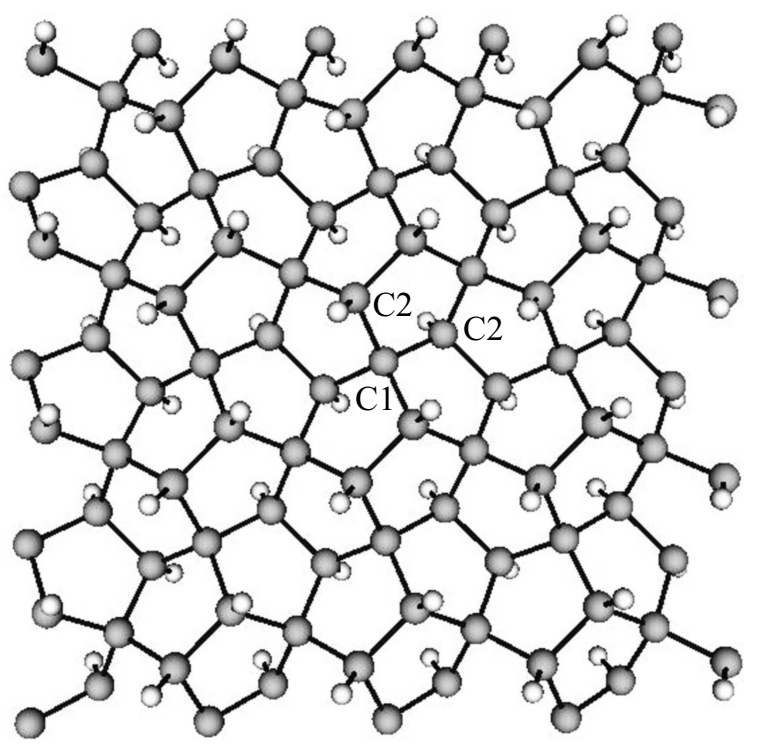

Рис. 2. Структура пентаграфана. Большие шарики - атомы углерода, маленькие - атомы водорода.

работы [13] делают вывод о возможности синтеза пентаграфана в условиях реального эксперимента.

Нужно, однако, иметь в виду, что существенное влияние на свойства пентаграфана может оказывать искажение его структуры из-за десорбции водорода. Основной целью настоящей работы является численное моделирование термоактивированной десорбции водорода из пентаграфана и анализ влияния десорбции на механические и электронные характеристики.

\section{2. Методы расчета}

Примитивная элементарная ячейка пентаграфана состоит из десяти атомов: шести атомов углерода и четырех атомов водорода, образующих связи $\mathrm{H}-\mathrm{C} 2$. Для моделирования мы использовали сверхъячейки $4 \times 4$ $\left(\mathrm{C}_{96} \mathrm{H}_{64}\right)$ и $6 \times 6\left(\mathrm{C}_{216} \mathrm{H}_{144}\right)$, состоящие из 16 и 36 примитивных ячеек соответственно. Граничные условия были периодическими в двух планарных направлениях и свободными в поперечном направлении. Периоды сверхъячеек с различным числом водородных вакансий всякий раз определялись из условия минимума энергии после релаксации по координатам всех атомов.

Межатомные взаимодействия $\mathrm{C}-\mathrm{C}, \mathrm{C}-\mathrm{H}$ и $\mathrm{H}-\mathrm{H}$ рассчитывались в рамках неортогональной модели сильной связи [15], которая учитывает валентные электроны всех атомов углерода и водорода. Эта модель хорошо зарекомендовала себя при численном моделировании различных углеродных и углеводородных наноструктур, включая графан, алмазоподобные углеродные нанонитки и пр. (см. работы $[11,16-20]$ и ссылки в них).

Моделирование временно́й эволюции сверхъячейки, нагретой до заданной температуры, проводилось сле- дующим образом. Действующие на атомы силы вычислялись по формуле Хельмана-Фейнмана. Затем уравнения движения Ньютона решались численно методом скоростей Верле с шагом по времени 0.3 фс. Для моделирования сверхъячейки с заданной концентрацией водородных вакансий мы случайным образом удаляли из нее соответствующее количество атомов водорода.

Спектры собственных колебаний атомов сверхъячейки вычислялись путем численной диагонализации соответствующей динамической матрицы. Эти спектры затем использовались для определения плотности фононных состояний (phonon density of states, PDOS).

\section{3. Результаты и их обсуждение}

Для длин межатомных связей мы получили следующие значения: $d(\mathrm{C} 1-\mathrm{C} 2)=1.55 \AA$ и $d(\mathrm{C} 2-\mathrm{C} 2)=$ $=1.53 \AA$, что совпадает с данными расчетов из первых принципов [13]. Таким образом, гидрирование пентаграфена приводит к существенному уменьшению разницы $\Delta d=d(\mathrm{C} 1-\mathrm{C} 2)-d(\mathrm{C} 2-\mathrm{C} 2)$ между длинами различных связей. В результате этого значительно ослабляются внутренние механические напряжения. В пентаграфане все атомы углерода находятся в состоянии $s p^{3}$-гибридизации.

\section{1. Температурная зависимость времени десорбции}

Характерное время начала десорбции мы полагали равным времени отрыва $\tau$ от сверхъячейки первого атома (или первой молекулы) водорода после нагревания сверхъячейки до заданной температуры $T$. Расчеты проводились при $T=(2000-3000)$ К. Выбор для моделирования столь высоких температур обусловлен тем, что при понижении температуры скорость протекания термоактивированных процессов (к которым относится и десорбция) уменьшается экспоненциально согласно закону Аррениуса:

$$
\tau^{-1}(T)=A \exp \left(-\frac{E_{a}}{k_{\mathrm{B}} T}\right)
$$

где $A-$ частотный фактор с размерностью $\mathrm{c}^{-1}, E_{a}-$ энергия активации данного процесса, $k_{\mathrm{B}}-$ постоянная Больцмана. Соответственно экспоненциально возрастает и компьютерное время, необходимое для такого „численного эксперимента“. Проведение вычислений при повышенных температурах позволяет непосредственно рассчитать зависимость $\tau(T)$ в заданном температурном диапазоне, а затем экстраполировать полученные результаты на область более низких температур.

Десорбцию молекулы $\mathrm{H}_{2}$ мы наблюдали только 3 раза. В остальных $\sim 50$ случаях имела место десорбция атомарного водорода. Расчетная зависимость $\tau$ от обратной температуры приведена на рис. 3 для сверхъячейки $4 \times 4$. Видно, что она в целом достаточно хорошо 


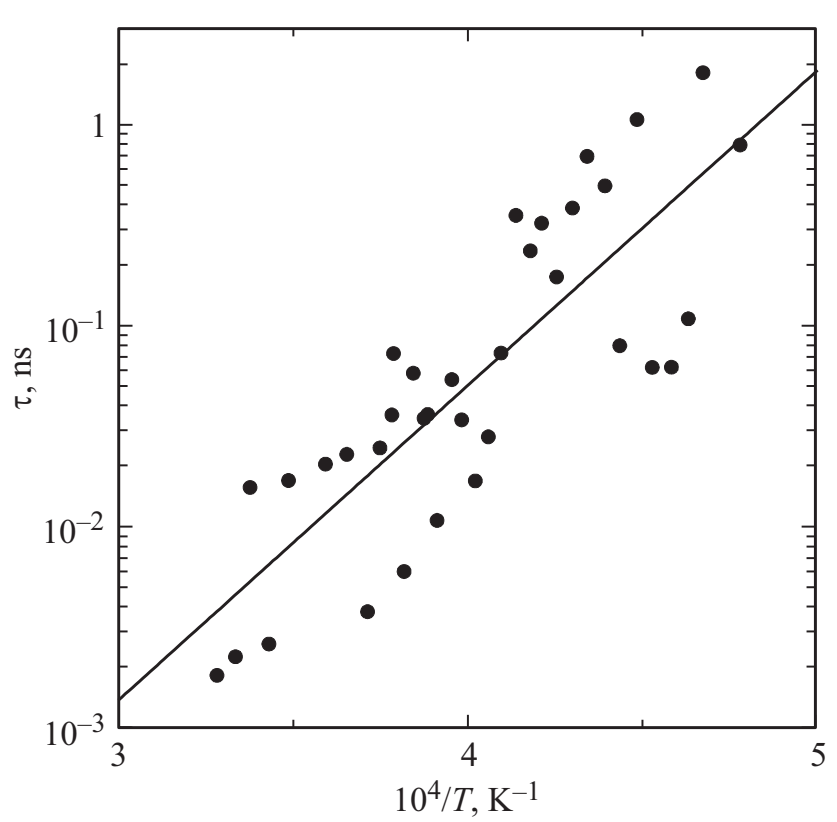

Рис. 3. Температурная зависимость времени $\tau$ десорбции водорода из пентаграфановой сверхъячейки $4 \times 4$. Точки - результаты расчета, сплошная линия - линейная аппроксимация методом наименьших квадратов.

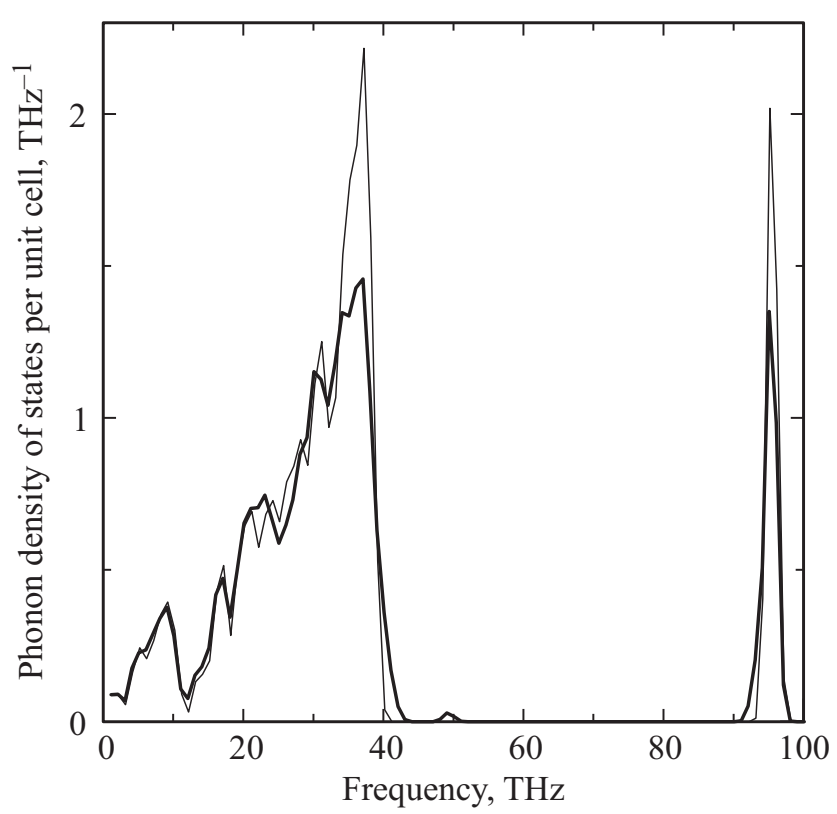

Pис. 4. Плотность фононных состояний пентаграфана для сверхъячейки $\mathrm{C}_{216} \mathrm{H}_{144}$ без водородных вакансий (тонкая линия) и сверхъячейки $\mathrm{C}_{216} \mathrm{H}_{116}$ с $20 \%$ вакансий (толстая линия).

описывается формулой Аррениуса (1). Большой разброс величины $\tau$ обусловлен вероятностной природой процесса десорбции. Статистический анализ полученных данных дает для энергии активации и частотного фактора этого процесса значения $E_{a}=(3.1 \pm 0.4)$ эВ и $A=(3.7 \pm 0.7) \cdot 10^{16} \mathrm{c}^{-1}$ соответственно. Например, при $T=1000 \mathrm{~K}$ из $(1)$ получим $\tau \sim 0.1$ с и т.п.

\section{2. Плотность фононных состояний}

При всех изученных нами значениях концентрации водородных вакансий спектры собственных колебаний соответствующих сверхъячеек не содержат мнимых частот, т.е. отвечают метастабильным атомным конфигурациям (локальным минимумам на поверхности потенциальной энергии).

На рис. 4 представлена PDOS сверхъячейки 6х6 без водородных вакансий. Максимумы PDOS при $f=37$ и 95 ТГц обусловлены преимущественно колебаниями атомов углерода и водорода соответственно. Наши результаты не сильно отличаются от данных расчетов из первых принципов, которые для частот этих максимумов дают значения около 40 и 80 ТГц [13].

При десорбции 10\% водорода положение пиков PDOS практически не изменяется, а их высота уменьшается на 15-20\%. После увеличения концентрации водородных вакансий до 20\% высота максимумов PDOS становится на 30-35\% меньше исходной (до десорбции) величины (см. рис. 4).

\section{3. Электронная структура}

Плотность электронных состояний для сверхъячейки $6 \times 6$ приведена на рис. 5, $a$, из которого видно, что пентаграфан является диэлектриком. Разность энергий нижней незаполненной (LUMO) и верхней заполненной (НОМО) молекулярных орбиталей составляет 5.60 эВ, что хорошо согласуется с шириной запрещенной зоны $E_{g}=5.78$ эВ, найденной в работе [13] путем более строгих расчетов.

При десорбции одного атома водорода вблизи дна запрещенной зоны возникает локальный уровень энергии (рис. 5,a). По мере дальнейшей десорбции число таких уровней увеличивается, и они формируют примесную зону, в которой находится уровень Ферми. Аналогичная картина имеет место при дегидрировании графана [20] и алмазоподобных углеродных нано ниток [18]. С ростом числа водородных вакансий ширина примесной зоны увеличивается, а плотность состояний на уровне Ферми остается отличной от нуля (рис. $5, b$ ), т. е. частично дегидрированный пентаграфан должен быть проводником.

\section{4. Механическая жесткость}

При анализе влияния десорбции водорода на механическую жесткость пентаграфана мы численно определяли упругие постоянные $\mathrm{C}_{11}, \mathrm{C}_{22}, \mathrm{C}_{12}$ сверхъячейки $6 \times 6$ с различным числом водородных вакансий и вычисляли модули Юнга для деформации вдоль осей $x$ и $y$ по формулам $E_{x}=\left(C_{11} \mathrm{C}_{22}-\mathrm{C}_{12}^{2}\right) / \mathrm{C}_{22}$ и $E_{y}=\left(\mathrm{C}_{11} \mathrm{C}_{22}-\mathrm{C}_{12}^{2}\right) / \mathrm{C}_{11}$ соответственно [21]. Для сверхъячейки без вакансий $E_{x}=E_{y}=219$ ГПа $\cdot$ нм, что близко к величине 209 ГПа · нм, полученной в работе [13] путем расчетов из первых принципов. 

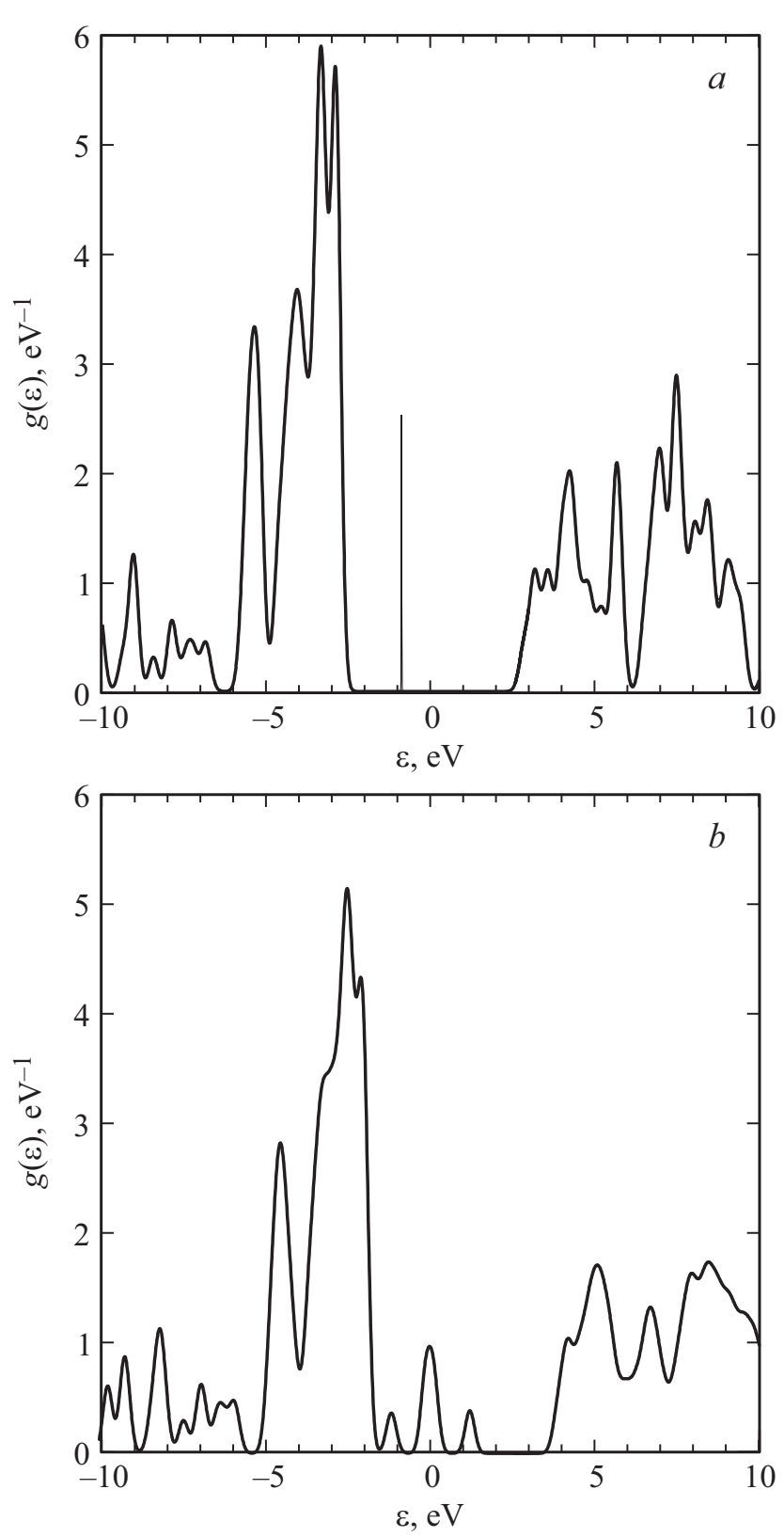

Рис. 5. Плотность электронных состояний пентаграфана $g(\varepsilon)$ в расчете на спин и на элементарную ячейку. За начало отсчета энергии принят уровень Ферми. $a$ : толстая линия для сверхъячейки $\mathrm{C}_{216} \mathrm{H}_{144}$ без водородных вакансий. Тонкая вертикальная линия - локальный энергетический уровень, появляющийся в сверхъячейке $\mathrm{C}_{216} \mathrm{H}_{143}$ при десорбции одного атома водорода. $b$ : для сверхъячейки $\mathrm{C}_{216} \mathrm{H}_{116}$ с $20 \%$ водородных вакансий.

В общем случае для данной конфигурации водородных вакансий величины $E_{x}$ и $E_{y}$ отличаются друг от друга, причем иногда весьма значительно. Мы не вычисляли средние по конфигурациям вакансий значения $E_{x}$ и $E_{y}$ по отдельности, а для каждой концентрации вакансий находили модуль Юнга $E$ как среднее не только по различным конфигурациям вакансий, но и по двум направлениям ( $x$ и $y$ ), подобно тому как мы делали это ранее для графана [20]. Мы обнаружили, что десорбция 10 и 20\% водорода приводит к уменьшению модуля Юнга до $E=(213 \pm 1)$ и $(209 \pm 1)$ ГПа соответственно. После полной десорбции водорода величина $E$ становится равной своему значению 252 ГПа · нм в пентаграфене [11]. Таким образом, зависимость модуля Юнга пентаграфана от содержания водорода оказывается немонотонной, как и в графане [20].

Заметим, что в пентаграфане (в отличие от пентаграфена) мы не наблюдали образования дефектов, появляющихся при неполной трансформации Стоуна-Уэльса повороте связи $\mathrm{C} 2-\mathrm{C} 2$ на угол $45^{\circ}-$ и инициирующих быстрое разрушение монослоя [11]. Это связано с тем, что, как показывают расчеты, энергия формирования таких дефектов в пентаграфане оказывается очень большой ( 9 эВ). Следовательно, в пентаграфане этот механизм разрушения структуры не работает, и основную роль в дефектообразовании играет термоактивированная десорбция водорода.

\section{4. Заключение}

1. Термоактивированная десорбция водорода является основной причиной разрушения структуры пентаграфана при нагревании.

2. По мере увеличения числа водородных вакансий в запрещенной энергетической зоне формируется примесная зона, в которой находится уровень Ферми.

3. Десорбция водорода приводит также к быстрому уменьшению максимумов плотности фононных состояний при $\sim 90$ и 40 ТГц.

4. При десорбции водорода модуль Юнга сначала уменьшается, а затем вновь возрастает до своего значения в пентаграфене.

Работа поддержана грантом РФФИ № 18-02-00278-а и выполнена при поддержке Министерства образования и науки РФ в рамках Программы повышения конкурентоспособности НИЯУ МИФИ.

\section{Список литературы}

[1] H.W. Kroto, J.R. Heath, S.C. O'Brien, R.F. Curl, R.E. Smalley. Nature, 318, 162 (1985).

[2] S. Iijima. Nature, 354, 56 (1991).

[3] K.S. Novoselov, A.K. Geim, S.V. Morozov, D. Jiang, Y. Zhang, S.V. Dubonos, I.V. Grigorieva, A.A. Firsov. Science, 306, 666 (2004).

[4] A.J. Stone, D.J. Wales. Chem. Phys. Lett., 128, 501 (1986).

[5] V. Georgakilas, J.A. Perman, J. Tucek, R. Zbori. Chem. Rev., 115, 4744 (2015).

[6] S. Zhang, J. Zhou, Q. Wang, X. Chen, Y. Kawazoe, P. Jena. Proc. Nat. Acad. Sci., 112, 2372 (2015).

[7] C.P. Ewels, X. Rocquefelte, H.W. Kroto, M.J. Rayson, P.R. Briddon, M.I. Heggie. Proc. Nat. Acad. Sci., 112, 15609 (2015).

[8] S. Winczewski, M.Y. Shaheen, J. Rybicki. Carbon, 126, 165 (2018). 
[9] H. Prinzbach, A. Weller, P. Landenberger, F. Wahl, J. Worth, L.T. Scott, M. Gelmont, D. Olevano, B. von Issendorff. Nature, 407, 60 (2000).

[10] И.В. Давыдов, А.И. Подливаев, Л.А. Опенов. ФТТ, 47, 751 (2005).

[11] Л.А. Опенов, А.И. Подливаев. Письма ЖЭТФ, 107, 747 (2018).

[12] P. Avramov, V. Demin, M. Luo, C.H. Choi, P.B. Sorokin, B. Yakobson, L. Chernozatonskii. J. Phys. Chem. Lett., 6, 4525 (2015).

[13] H. Einollahzadeh, S.M. Fazeli, R.S. Dariani. Sci. Technol. Adv. Mater., 17, 610 (2016).

[14] J.O. Sofo, A.S. Chaudhari, G.D. Barber. Phys. Rev. B, 75, 153401 (2007)

[15] M.M. Maslov, A.I. Podlivaev, K.P. Katin. Molecular Simulation, 42, 305 (2016).

[16] K.P. Katin, V.S. Prudkovskiy, M.M. Maslov. Physica E, 81, 1 (2016).

[17] А.И. Подливаев, Л.А. Опенов. ФТП, 51, 222 (2017).

[18] А.И. Подливаев, Л.А. Опенов. ФТП, 51, 667 (2017).

[19] M.M. Maslov, K.P. Katin. Chem. Phys. Lett., 644, 280 (2016).

[20] Л.А. Опенов, А.И. Подливаев. ФТП, 52, 614 (2018).

[21] R.C. Andrew, R.E. Mapasha, A.M. Ukpong, N. Chetty. Phys. Rev. B, 85, 125428 (2012).

Редактор А.Н. Смирнов

\section{Hydrogen desorption from pentagraphane}

\section{L.A. Openov, A.I. Podlivaev}

\section{National Research Nuclear University „MEPhl“, 115409 Moscow, Russua}

Abstract By means of atomistic computer sumulation we study the thermally activated desorption of hydrogen from pentagrafane, a recently predicted quasi-twe-dimensional hydrocarbon compound, which is a pentagrafene monolayer coated on both sides by hydrogen, in which the $\mathrm{C}-\mathrm{C}$ bonds form a grid of adjacent pentagons, and characteristic of carbon nanostructures hexagons are absent. The effect of hydrogen desorption on the electronic structure, the density of phonon states, and Yong's modulus is studied. The temperature dependence of the characteristic desorption time is determined by molecular dynaics method. 